\title{
Electric properties of metal-organic lyotropic liquid crystals
}

\author{
G.V. Klimusheva, A.V. Koval'chuk \\ Institute of Physics, NAS of Ukraine, 46 prospect Nauky, 03028 Kiev, Ukraine \\ E-mail:klimush@iop.kiev.ua
}

\begin{abstract}
The electric properties of pure and dye-doped potassium caproate, which in a 1:1 water solution forms at room temperature ionic lyotropic liquid crystals (ILLC) of smectic $A$ type [1], were investigated. It carried out the comparative analysis of electric properties of the investigated objects with other molecular liquid crystals (LC). It was established, that maximal field strength (at voltages $U<2 \mathrm{~V}$ ) in ILLC, as in the case of usual LC, takes place near the electrodes, where the double electric layer (DEL) is formed. The charge transfer in this region can be described by model of the Schottky emission through a thin dielectric layer. Introducing $0.01 \mathrm{wt} . \%$ of ionic polymethine dye [2,3] in ILLC results in insignificant rise of conductivity of samples and increase of thickness of DEL. For samples with $\mathrm{Cu}$ and covered with polyimide ITO electrodes cyclic voltage-current characteristics were obtained at different voltage polarity. The ratio of concentration of charge carriers with different signs is estimated.
\end{abstract}

Keywords: ionic lyotropic liquid crystals, near-electrode processes, conductivity, charge exchange.

Paper received 25.02.03; accepted for publication 16.06.03.

\section{Introduction}

In many cases, researches of electric properties of liquid crystals require to take into account processes that occur at electrode-LC interface. The conductivity of an electrode is electronic while that of LC is ionic. In this case, a question about charge exchange mechanism on the electrode-LC boundary arises. It is known [4] that in organic liquids with a large concentration of ions (electrolytes) the exchange of charges at the boundary occurs due to the direct contact of charge carriers with the electrode by redox processes. For long and a priori such mechanism of charge transport has been considered as the basic one at the analysis of electric properties of liquid crystals. It was recently shown [5] that at the voltage lower than $1 \mathrm{~V}$ an exchange of charges between the electrode and ions of molecular LC is more complicated due to the presence of the neutral molecules. The concentration of the neutral molecules in LC is much higher than the concentration of ions. Therefore, adsorption of ions results in the formation of a dielectric layer, basic part of which consists of the neutral molecules [5,6]. This layer forms a buffer zone between an electrode and mobile ions and defines the mechanism of a charge transfer at low voltages.
The tunnel mechanism of charge transfer (Schottky emission through dielectric layer) leads to a long process of dielectric relaxation (tens, hundreds of seconds and more). As it was shown in [7], the time of such low-frequency relaxation is proportional to the resistivity of the substance. In this case the time of the charge redistribution at DEL becomes comparable with the time of charge exchange in LC-electrode region.

One can expect that it is possible to distinguish partially the low-frequency near-electrode processes and electrochemical processes of charge exchange in ILLC, which is considerably more conductive than usual LC.

The purpose of present work is to investigate whether the dielectric layer in ILLC forms and whether it influences on the conductivity of a sample, as well as to investigate the redox processes on the boundary of electrodeILLC.

\section{Materials and methods}

The electric properties of pure ILLC and ILLC with an impurity of polymethine dye (DILLC) [2,3] were investigated. The structural formula of the dye molecule is given in Fig. 1. The samples had a sandwich-like structure com- 
<smiles>CCCCCCC(C#N)C=CC=CC=C(C#N)C#N</smiles>

Fig. 1. Structure formula of the dye molecule.

prising glass substrates covered with the transparent ITO electrodes. The thickness of the samples in the range of 20-50 $\mu \mathrm{m}$ was set by teflon stripes placed on a security electrodes. The measurements were carried out at the temperature $293 \mathrm{~K}$. The electric properties of samples were investigated applying a stationary as well as a variable voltage.

In the case of a stationary voltage, a long-lasting (tens of minutes) decay of the current was observed in ILLC, like to that of usual LC. The dependence of a steadystate current density $J_{S T}$ on voltage $U$ was investigated. For the voltage range from 0.01 up to $5 \mathrm{~V}$ the values of $J_{S T}$ and the maximal reverse current density $J_{R M}$ were determined. For this purpose, sample, in which the current has reached the steady-state value, was shortcircuited by the measuring device. The transient characteristics of the reverse current were studied according to the technique offered in [8]. The maximal value of polarization voltage $U_{P}$, the conductivity of the direct current $\sigma_{D C}$, diffusion coefficient $D$ and mobility of charge carriers $\mu$ were determined.

In the case of variable voltage the values of resistance $R$ and capacity $C$ in the frequency range from $10^{-2}$ to $10^{6}$ $\mathrm{Hz}$ were determined by means of oscilloscopic method $[9,10]$ with the amplitude of the measuring signal $0.25 \mathrm{~V}$. The dielectric permittivity $\varepsilon$ and conductivity of the alternating current $\sigma_{A C}$ were determined in the frequency range, where the dispersion of $R$ and $C$ was not observed.

The features of the electrochemical processes in a sample were analyzed by means of cyclic voltage-current measurements, similar to [4]. Although these measurements did not result in exact determination of redox potentials, they provide an opportunity to analyse mechanisms of charge transfer and charge exchange in ILLC. The copper and covered with polyimide layer ITO (Pelectrode) electrodes were used. Linearly varied voltage was applied to the sample. The peak value of the voltage was in the range of 1.5-2.5 V. The rate of the voltage rise and decrease was constant within an experimental run. For various experiments it was varied from 0.3 to $300 \mathrm{mV} / \mathrm{s}$.

\section{Experiment and discussion}

As it follows from experimental data, the application of a stationary voltage to the samples, irrespective to its value, results in an essential decreasing of current value (more than by two orders of magnitude). The current kinetics shows the charge redistribution in the near-electrode region and the formation of a double electric layer with polarization voltage $U_{p}(t)$ at given instant of time $t$, which directed against external field. Assuming that the volume resistance of a sample $R_{V}$ does not depend on the applied voltage $U$ (that is proved by experimental data), the current kinetics could be described by a rather simple ratio

$$
J=\frac{U-U_{P}(t)}{R_{V} S},
$$

where $S$ is an area of electrodes. As the steady-state value of the current corresponds to the saturated value of $U_{p}(t)=U_{p}$, then

$$
J_{S T}=\frac{U-U_{P}}{R_{V} S} \equiv \frac{U_{R}}{R_{V} S} .
$$

Polarization voltage causes the reverse current when the external field is switched off. It maximal value is equal to

$$
J_{R M}=\frac{U_{P}}{R_{V} S} .
$$

From equations (2) and (3) follows, that

$$
\frac{U_{P}}{U}=\frac{J_{R M}}{J_{R M}+J_{S T}} .
$$

The dependence $U_{P} / U$ on $U$ for the sample ITOILLC-ITO is presented in Fig. 2. From the data obtained follows, that almost whole internal electric field is enclosed at the near-electrode region at low external voltage $(U<2 \mathrm{~V})$. The same dependence $U_{P / U}$ on $U$ was obtained for the sample ITO-DILLC-ITO. The only difference observed is the voltage, at which this ratio sharply decreases. For DILLC it takes place at $U<1.5 \mathrm{~V}$. Thus, for both ILLC and DILLC the current flow through the

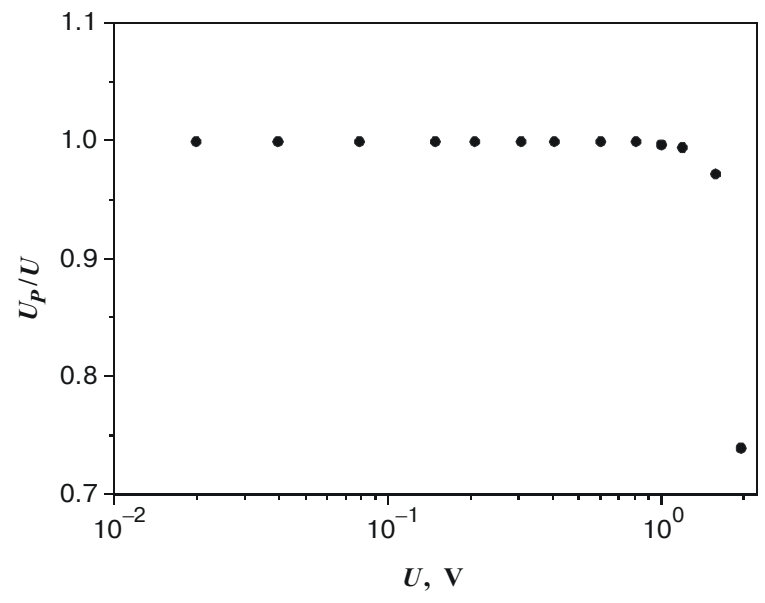

Fig. 2. Dependence of $U_{P} / U$ ratio on voltage $U$ of the sample ITO-ILLC-ITO. Thickness of the sample is $20 \mu \mathrm{m}$. Temperature is 293 K.

SQO, 6(2), 2003 


\section{G.V. Klimusheva et al.: Electric properties of metal-organic lyotropic liquid ...}

cell is determined by the boundary properties at low voltages. The mechanism of the current flow through such a boundary is possible to figure out on the basis of the analysis of the voltage-current $(J-V)$ characteristics of the steady-state current $J_{S T}$. As in case of molecular LC [5], various models were used for the analysis of $J-V$ characteristics. It was found that the least deviation of the experimental points from a straight line is observed in coordinates $\lg J_{S T}$ on $U^{1 / 2}$ (see Fig. 3). As it was shown in $[5,11]$ such mechanism of current flow is the Schottky emission through a dielectric layer, which is described by a ratio

$$
J=J_{0} \exp \left(\alpha U^{1 / 2}\right),
$$

where $J_{0}$ and $\alpha$ are parameters, which depend on external conditions as well as on properties of substance.

If almost all voltage is enclosed to near-electrode region at $U<1.5 \mathrm{~V}$, then

$U=E d_{d}$,

where $E$ is the electric field strength, $d_{d}$ is the thickness of the dielectric layer. According to [11] $a$ is equal

$\alpha=\frac{e}{k T}\left(\frac{e}{4 \pi \varepsilon \varepsilon_{0} d_{d}}\right)^{1 / 2}$.

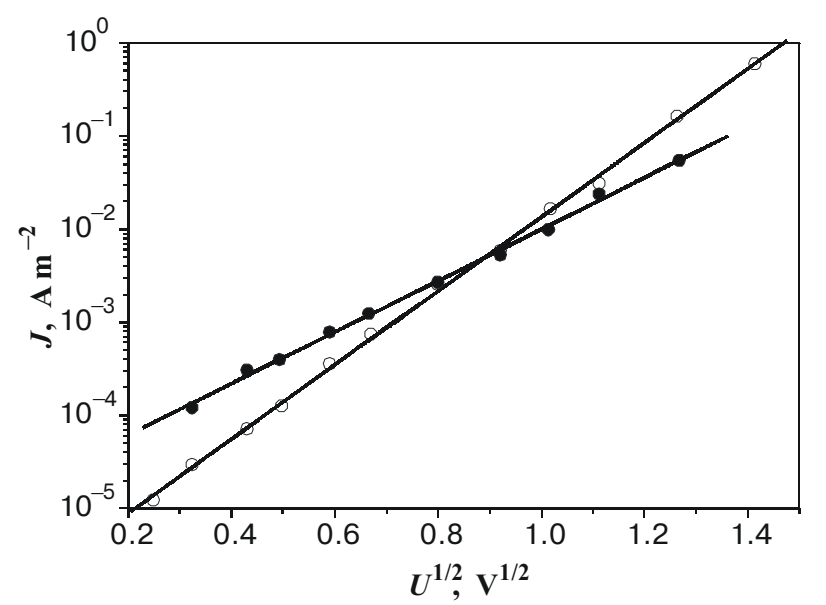

Fig. 3. Voltage-current characteristics of the samples ITO-ILLCITO (open points) and ITO-DILLC-ITO (solid points). Thickness of the samples is $20 \mu \mathrm{m}$. Temperature is $293 \mathrm{~K}$.
Here $e$ is electron charge, $k$ is Boltzmann constant, $\varepsilon_{0}$ is dielectric permittivity of vacuum, $\varepsilon$ is dielectric permittivity of substance.

The analysis of dependence of $R$ and $C$ on frequency shows, that for $f>5 \cdot 10^{4} \mathrm{~Hz}$ the dispersion of these parameters is not observed. Therefore it is possible to accept, that these values characterize bulk properties of substance. The values of $e$ and $\sigma_{A C}$ for ILLC and DILLC are listed in the Table. Obviously, the addition of an impurity to ILLC does not practically change value e and insignificantly increases $\sigma_{A C}$.

In the Table values $d_{d}$ calculated using ratio (7) are given as well. Comparison of values $d_{d}$ of ILLC with $d_{d}$ of LC obtained in [5,6] shows, that they almost coincide, though $\sigma_{A C}$ in ILLC and LC differ more than by three orders of magnitude. In pure and dye-doped ILLC thin dielectric layer is also caused by presence of neutral molecules.

From the $J-V$ characteristics at $U>2 \mathrm{~V}$ follows that the linear dependence of the current on the voltage is peculiar for ILLC and DILLC. The same $J$ - $V$ dependence was observed previously for molecular LC [5]. The conductivity of direct current $\sigma_{D C}$ was obtained when the voltage $U_{R}$ (see Eq. (2)) was enclosed to a sample bulk. From the Table follows that the value $\sigma_{D C}$ is less then $\sigma_{A C}$, though the ratios of conductivity values at application of direct or alternating current for ILLC and DILLC coincide practically.

The comparison of conductivity data shows, that it for the ILLC is higher by three orders of magnitude than that for the LC. It was interesting to investigate, whether such difference is connected with difference of mobility of charges or with difference of concentration of charge carriers. The measurements of transitive $J$ - $V$ characteristics using a technique offered in [13] have shown, that at different values of voltage and ratio of polarization/ pulling voltages a maximum of current is not observed. Consequently, the dependence of the released charge $Q$ on time $t$ was analysed on a basis of $J_{R M}$ kinetics $(U>2 \mathrm{~V})$ obtained by short-circuiting of ILLC cell on the measuring device. Similar to [8], the $Q(t)$ dependence observed is exponential. It is allow us to use for the definition of charge diffusion coefficient a following relationship

$$
D=\frac{\left(\ln Q_{1}-\ln Q_{2}\right) d^{2}}{\pi^{2}\left(t_{2}-t_{1}\right)},
$$

where $Q_{1}$ and $Q_{2}$ are released charges from a cell through time $t_{1}$ and $t_{2}$, respectively. The values $D$ of ILLC and

Table. The volume and near-electrode parameters describing electric properties of pure ILLC and ILLC with 0.01 wt. \% polymethine dye (DILLC). Thickness of samples is $20 \mu \mathrm{m}$. Temperature is $293 \mathrm{~K}$.

\begin{tabular}{llllllll}
\hline \hline Sample & $\varepsilon$ & $\sigma_{A C}, \mathrm{Om}^{-1} \mathrm{~m}^{-1}$ & $\sigma_{D C}, \mathrm{Om}^{-1} \mathrm{~m}^{-1}$ & $d_{d}, \mathrm{~nm}$ & $D, \mathrm{~m}^{2} \mathrm{~s}^{-1}$ & $\mu, \mathrm{m}^{2} \mathrm{~V}^{-1} \mathrm{~s}^{-1}$ & $n_{A C}, \mathrm{~m}^{-3}$ \\
\hline ILLC & 7.5 & $5.0 \cdot 10^{-4}$ & $9.0 \cdot 10^{-5}$ & 4.4 & $1.2 \cdot 10^{-11}$ & $4.8 \cdot 10^{-10}$ & $6.5 \cdot 10^{24}$ \\
\hline DILLC & 7.8 & $7.2 \cdot 10^{-4}$ & $1.2 \cdot 10^{-4}$ & 7.2 & $1.1 \cdot 10^{-11}$ & $4.4 \cdot 10^{-10}$ & $1.0 \cdot 10^{25}$ \\
\hline \hline
\end{tabular}




\section{G.V. Klimusheva et al.: Electric properties of metal-organic lyotropic liquid ...}

DILLC are given in the Table. It is easy to see, that the diffusion coefficients as well as conductivity of pure and dye-doped ILLC differ insignificantly.

On the basis of the Einstein equation and determined diffusion coefficient $D$ (see Eq. (8)) the value of charge mobility $\mu$ was evaluated as

$$
\mu=D \frac{e}{k T} \text {. }
$$

The concentration of carriers of the charge $n$ was defined from well-known relationship for conductivity. These data are given in the Table. The comparison of parameters obtained with corresponding values for molecular LC shows, that the difference in conductivity of these substances is basically caused by essential difference of value $n$ (more than $10^{3}$ time).

For division of oxidation and reduction processes in ILLC the cyclic voltamperogrames were measured using cells with different electrodes. It was supposed that the electrochemical processes on boundary of P-electrode would be insignificant because it covered with polyimide layer and the basic contribution to current value will cause the charge exchange near $\mathrm{Cu}$-electrode. As it follows from Fig. 4 the voltage applied to the cell is strongly asymmetric relatively to the potential of "ground" (voltage applied to the cell had one polarity during a significant period of time and another polarity during a short period of time). Those polarities will be referred bellow at which the cell stays for a long period of time although the measurements were carried out at different polarity on each of electrodes.

In a Fig. 4a the cyclic voltamperogram is given for cell "+" Cu-ILLC-P "-" with rate of voltage change $1 \mathrm{mV} / \mathrm{s}$. One could see that at increasing of voltage the small current maximum is observed in the region of $U=$ $=0.015 \mathrm{~V}$ (here and further voltage is given relatively to zero ground potential). This maximum is looking clearer with the decreasing of the value $v$. The current caused recharge of DEL becomes essential at $v>1 \mathrm{mV} / \mathrm{s}$. There- fore the current maximum was not practically observed on cyclic voltamperogram at the rate of $10 \mathrm{mV} / \mathrm{s}$.

In a Fig. 4b cyclic voltamperogram is given for cell "-" Cu-ILLC-P "+" at the same value $v(1 \mathrm{mV} / \mathrm{s})$. In this case one current maximum is observed at increasing of voltage and two current maxima at decreasing of a voltage, however, at more significant values $U$, than for data given on Fig. 4a. Increasing of rate $v$ results in practically disappearing of the current maxima on cavity site of voltage decrease. The current maximum occurring on site of voltage increase was rather clearly observed down to $v=50 \mathrm{mV} / \mathrm{s}$.

On the basis of data obtained and assumption, that the electrochemical processes occur mainly near the $\mathrm{Cu}$ electrode, it is possible to conclude, that the current maximum in Fig. 4a corresponds to process of oxidation, while the maximum in Fig. 4b corresponds to process of reduction. Then from a relationship for a pike current [4]

$J=\beta z^{2 / 3} D^{1 / 2} C v^{1 / 2}$,

(where $\beta$ is Rendlz-Shevchuk constant, $z$ is number of electrons, participating in electrochemical reaction and $C_{ \pm}$is the concentration of ions) it is possible to found concentration ratio of positive $C_{+}$and negative $C_{-}$ions. Suppose, that $D_{+}=D_{-}$, then

$\frac{J_{a}}{J_{b}}=\frac{C_{-}}{C_{+}}$.

From data obtained follows, that $\mathrm{C}_{-} / C_{+} \approx 3 \cdot 10^{-2}$, i.e. the charge transfer in ILLC is carried out by the positively charged ions. According to a model offered in [5], the asymmetry of concentration of charge carriers of different signs not necessarily should cause asymmetry of conductivity relatively to polarity of the voltage applied. As the strong enough electric fields have place near electrodes, especially near the anode, the ions, which passed to neutral state, could be recharged. Such recharge of ions can be that basic reason that causes the asymmetry in the concentration of charge carriers.
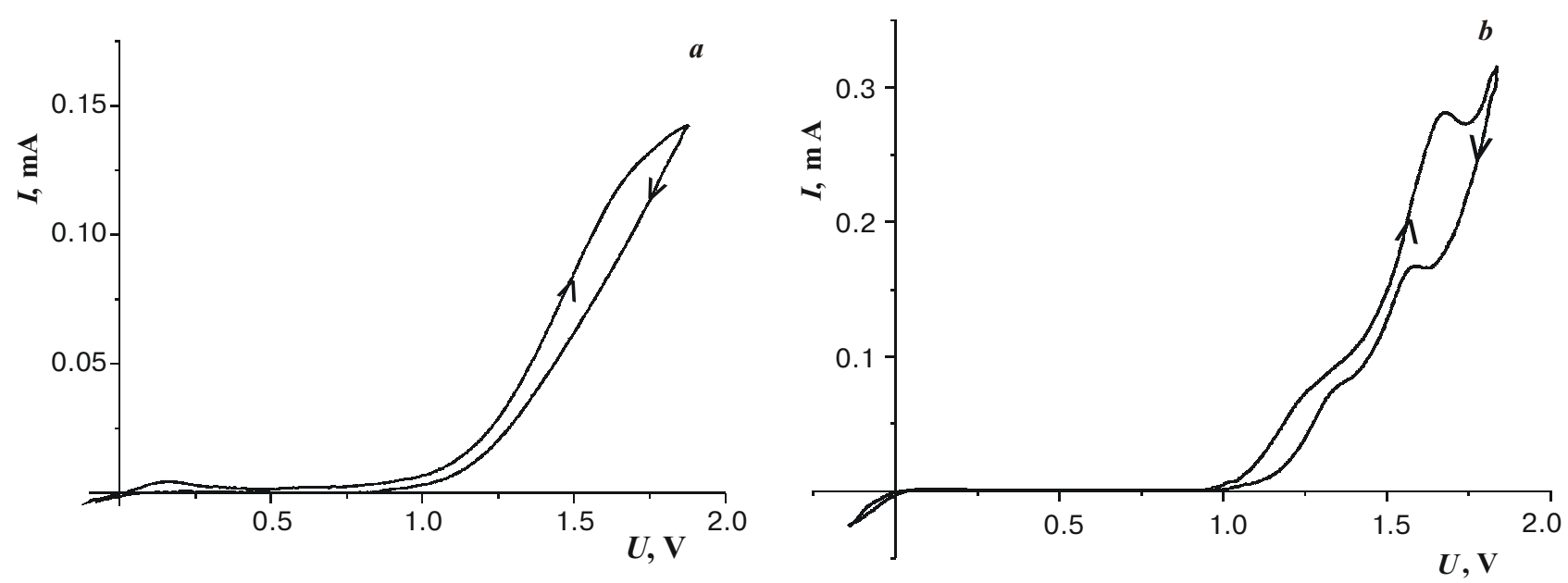

Fig. 4. Cyclic voltamperogrames of the sample "+" Cu-ILLC-P "-" at positive (a) and negative (b) polarity of Cu-electrode. Rate of the voltage change is $1 \mathrm{mV} / \mathrm{s}$. The thickness of the sample is $45 \mu \mathrm{m}$. The temperature is $273 \mathrm{~K}$. 


\section{G.V. Klimusheva et al.: Electric properties of metal-organic lyotropic liquid ...}

\section{Conclusions}

Based on the analysis of $J-V$ characteristics of steadystate current it was for the first time shown that in ILLC and DILLC as well as in the case of molecular LC, at $U<$ $1.5 \mathrm{~V}$, practically all external voltage is enclosed to near electrode layers.

The transfer of charge carriers through boundary of ILLC-electrode is described by the Shottky emission through thin dielectric layer. It was determined that the thickness of such layer in ILLC amounts to $4.4 \mathrm{~nm}$ and in DILLC amounts to $7.4 \mathrm{~nm}$. According to a model offered in [4], dielectric layer is formed due to adsorption of both ions and neutral molecules on to the electrode.

Using the analysis of the maximal reverse current kinetics, the charge diffusion coefficient and the mobility of charge carriers in ILLC and DILLC are determined. It is shown that these characteristics as well as the conductivity of current really do not change at introducing of dye into ILLC. The comparison of conductivity values of ILLC and LC shows that their difference (more than $10^{3}$ times) is basically caused by a difference in concentration of charge carriers.

It was for the first time observed the current maxima on cyclic voltamperogrames in ILLC cells with different electrodes. It is shown that the position and height of current maximum depend on polarity of $\mathrm{Cu}$-electrode near which an exchange of charges mainly takes place. The ratio of concentration of charges with different signs is estimated $\left.(\mathrm{C}\lrcorner \mathrm{C}_{+} \approx 3 \cdot 10^{-2}\right)$. Such difference in concentration of charge carriers can be caused by change of ions polarity near the anode where, according to model offered in [5], a basic part of barrier governing the exchange of charge carriers is disposed.

\section{References}

1. T.A. Mirnaya, V.D. Prisyazhnyi, V.A Shcherbakov, Uspekhi Khimii 58, 1429 (1989)

2. A.A. Ishchenko, Structure and Spectral Luminescent Properties of Polymethine Dyes (Naukova Dumka, Kiev.1994).

3. N. Volynets, N. Derevyanko, A.A. Ishchenko, G.V. Klimusheva, T.A. Mirnaya G. Yaremchuk, L.P. Yatsenko, Proc. SPIE, 4418, 36 (2001).

4. M.M. Baizer (Ed.), Organic Electrochemistry (Marcel Dekker, New York,1973).

5. A.V. Koval'chuk, JETP Letters 72, 377 (2000).

6. A.V. Koval'chuk, Ukr. Fiz. Zh. 46, 316 (2001).

7. A.V. Koval'chuk, Funct. Mat. 5 ,426 (1998).

8. N.I. Grisenko, N.V. Moshel, Ukr. Fiz. Zh. 25 ,1830 (1980).

9. F.J. Twarowski, A.C. Albrecht, J. Chem. Phys. 20, 2255 (1980).

10. A.V. Koval'chuk, Ukr. Fiz. Zh. 41, 991 (1996).

11. S.M. Sze, Physics of Semiconductor Devices (Wiley Interscience, New York, 1981). 